\title{
USO DE ADUBO DE LIBERAÇÃO LENTA E CONVENCIONAL NO CULTIVO DE Impatiens balsamina
}

\author{
Jéssica Cristina Meira Bezerra ${ }^{1 *}$, Patrick Luan Ferreira Santos ${ }^{2}$, Walter Aparecido Ribeiro \\ Júnior $^{3}$, Luiz Henrique Silvério Junior ${ }^{4}$, Regina Maria Monteiro de Castilho ${ }^{5}$.
}

\footnotetext{
${ }^{1}$ Graduando, Engenharia Agronômica, Universidade Estadual Paulista (UNESP), Faculdade de Engenharia, Ilha Solteira, SP. *E-mail do autor correspondente: jessicabezerra@unesp.br

${ }^{2}$ Doutorando, Engenharia Agronômica, Universidade Estadual Paulista (UNESP), Botucatu, SP.

${ }^{3}$ Doutorando, Engenharia Agronômica, Universidade Estadual de Londrina (UEL), Londrina, PR.

${ }^{4}$ Graduando, Engenharia Agronômica, Universidade Estadual Paulista (UNESP), Botucatu, SP.

${ }^{5}$ Docente, Engenharia Agronômica, Universidade Estadual Paulista (UNESP), Faculdade de Engenharia, Ilha Solteira, SP.
}

Recebido: 30/04/2020; Aceito: 09/02/2021

RESUMO: A Impatiens balsamina (beijo de frade, maria sem vergonha) é uma florífera ornamental, usada em vaso e para formação de canteiros; possui flores de cores vermelha, creme, laranja, rósea, branca, roxa ou mesclada, caule suculento e ereto, de altura variando entre 30 a $90 \mathrm{~cm}$, sendo tipicamente tropical. A pesquisa foi realizada com o intuito de avaliar a produção I. balsamina (Blue Line Flores / Balsamina Camélia Sortida) com uso de adubo de liberação lenta e convencional, em casa de vegetação tipo PAD\&FAN na UNESP /Ilha Solteira - SP. Jardineiras de polipropileno preto $(47,5 \mathrm{~cm} \mathrm{x} 17,5 \mathrm{~cm}$ boca; $41,5 \mathrm{~cm} \times 113 \mathrm{~cm}$ fundo e 15,5 cm altura: 8,5 litros), foram preenchidas com os tratamentos: T1- Condicionador Floreira + Forth Plantio $\left(13-5\right.$ - 13) $\left(5 \mathrm{~g} \mathrm{~L}^{-1}\right), \mathrm{T} 2$ - Condicionador Floreira + Osmocote ${ }^{\circledR} 3 \mathrm{M}$ 14-14-14 (6 g L $\left.{ }^{-1}\right)$ e T3 - Condicionador Floreira + Osmocote ${ }^{\circledR} 3 \mathrm{M} 19-06-10$ mini prill $(6 \mathrm{~g} \mathrm{~L}-$ ${ }^{1}$ ), e posteriormente transplantadas 2 mudas (mudas com 30 dias). Conclui-se que os melhores resultados foram obtidos com uso de adubo de liberação lenta, com ênfase no tratamento T3 - Substato + Osmocote ${ }^{\circledR} 3 \mathrm{M}$ 19-06-10 mini prill $\left(6 \mathrm{~g} \mathrm{~L}^{-1}\right)$.

Palavras-chave: Adubação. Beijo de frade. Planta ornamental. Fertilizante de eficiência aprimorada.

\section{USE OF SLOW AND CONVENTIONAL FERTILIZER IN THE CULTIVATION OF Impatiens balsamina}

\begin{abstract}
Impatiens balsamina (garden balsam, rose balsam) is an ornamental plant, used for potted and flower bed formation; it has flowers of red, cream, orange, pink, white, purple or mixed colors, succulent and erect stem, with height varying between 30 to $90 \mathrm{~cm}$, being typically tropical. The research was carried out in order to evaluate the production $I$. balsamina (Blue Line Flores / Balsamina Camélia Sortida) using slow and conventional release fertilizer, in a PAD\&FAN greenhouse at UNESP / Ilha Solteira - SP. Black polypropylene planters $(47.5 \mathrm{~cm} \times 17.5 \mathrm{~cm}$ mouth; $41.5 \mathrm{~cm} \times 113 \mathrm{~cm}$ bottom and $15.5 \mathrm{~cm}$ height: 8.5 liters), were filled with the treatments: T1- Floreira Conditioner + Forth Plantio $\left(13-5\right.$ - 13) $\left(5 \mathrm{~g} \mathrm{~L}^{-1}\right), \mathrm{T} 2$ - Floreira Conditioner + Osmocote $\AA 3 \mathrm{M} 14-14-14\left(6 \mathrm{~g} \mathrm{~L}^{-1}\right)$ and $\mathrm{T} 3$ -
\end{abstract}


Floreira Conditioner + Osmocote ${ }^{\circledR} 3 \mathrm{M}$ 19-06-10 mini prill $\left(6 \mathrm{~g} \mathrm{~L}^{-1}\right)$, and subsequently transplanted 2 seedlings (seedlings with 30 days). It was concluded that better results were obtained with the use of slow-release fertilizer, with emphasis on the treatment T3 - Substrate + Osmocote ${ }^{\circledR}$ 3M 19-06-10 mini prill $\left(6 \mathrm{~g} \mathrm{~L}^{-1}\right)$.

Key words: Fertilizer. Garden balsam. Ornamental plant. Enhanced efficiency fertilizers.

\section{INTRODUÇÃO}

O setor de flores e plantas ornamentais tem tido grande avanço no mercado nacional, sendo um dos segmentos do agronegócio que mais cresce na atualidade (MOREIRA; BENTO, 2018). Em 2018, o crescimento da produção de flores que chegou a $8 \%$ em relação a 2017, alcançando cerca de $\mathrm{R} \$ 8$ bilhões de faturamento de mercado, como previsto por Rivas (2018). De acordo com Brainer (2018) houve também a geração de 215,8 mil empregos diretos distribuídos entre a produção $(36,3 \%)$, atacado $(3,9 \%)$, varejo $(55,9 \%)$ e apoio $(3,9 \%)$.

Ainda, mostrando a atual importância da Floricultura, a Comissão de Agricultura e Reforma Agrária (CRA) do Senado aprovou, em 17/12/2019, o Projeto de Lei ${ }^{\circ} 4485$ que cria a Política Nacional de Incentivo à Cultura de Flores e de Plantas Ornamentais de Qualidade (BRASIL, 2020).

A floricultura possui diversos aspectos de produção, como plantas para vaso e para paisagismo, onde se enquadra a Impatiens balsamina, popularmente conhecida como beijo de frade, maria sem vergonha ou balsamina, que é caracterizada, segundo Alves (2019), por atingir entre 20 e $75 \mathrm{~cm}$ de altura, sendo que sua flor é pequena e tem entre 2 e $5 \mathrm{~cm}$ de diâmetro, com cores diversas, tanto em tonalidades únicas (branca, vermelha, creme, laranja e rosa), como também mescladas. É recomendada para áreas cuja condição se caracteriza sombreadas durante a metade do dia (planta de meia sombra) (TANAKA; CHAVES, 2019). Segundo Agristar (2020), é excelente para plantio em canteiros, jardineiras e vasos, e possui fácil adaptação às condições brasileiras.

De acordo com Furtini Neto, Boldrin e Mattson (2015), a qualidade visual de plantas ornamentais está intrinsecamente atrelada a um balanço adequado de nutrientes. A altura das plantas, forma e coloração são aspectos qualitativos de espécies ornamentais, influenciados pela nutrição mineral, dentre outros aspectos ambientais. Assim, na produção de plantas de qualidade deve-se considerar o tipo de substrato a ser utilizado, pois este fator afeta diretamente o desenvolvimento e a arquitetura do sistema radicular (LATIMER, 1991) e, para aumentar a eficácia, podem ser utilizados o emprego de adubos, de liberação lenta ou não.

A premissa básica para o uso dos adubos de liberação lenta é disponibilizar continuamente os nutrientes, reduzindo a possibilidade de perdas por lixiviação e mantendo a planta nutrida durante um maior período de crescimento (SHARMA, 1979). Trabalhando com produção de pimenta ornamental (Capsicum annuum) e doses de adubação com fertilizantes de liberação lenta e convencional, Backes et al. (2007) concluíram que o adubo de liberação lenta foi mais eficiente que o adubo convencional para a maioria das variáveis analisadas. 
Assim, o intuito desta pesquisa foi avaliar o uso de adubos por meio de análises de índice de clorofila foliar, altura, massa fresca e seca da parte aérea e da raiz e macro (N; P; K; $\mathrm{S}$; $\mathrm{Mg} ; \mathrm{Ca})$ e micronutrientes $(\mathrm{Cu} ; \mathrm{Fe} ; \mathrm{Mn})$, para produção de I. balsamina em Ilha Solteira SP.

\section{MATERIAL E MÉTODOS}

O experimento foi conduzido em casa de vegetação (PAD\&FAN), com temperatura média e umidade relativa controladas a $25^{\circ} \mathrm{C} \pm 3^{\circ} \mathrm{C}$ e $60 \%$ respectivamente, na UNESP / Campus de Ilha Solteira (latitude $20^{\circ} 25^{\prime} 24^{\prime}$ 'S, longitude 51 $21^{\prime} 13^{\prime}$ ' O e altitude de $337 \mathrm{~m}$ ), em Ilha Solteira - SP, no período de 24 de julho a 13 de setembro de 2017.

Foram utilizadas sementes Blue Line Flores / Balsamina Camélia Sortida ( $I$. balsamina), cedidas pela empresa TOPSEED ${ }^{\circledR}$, com as quais foram produzidas mudas, e transplantadas aos 30 dias após germinação, em jardineiras de polipropileno preto $(47,5 \mathrm{~cm} \mathrm{x}$ $17,5 \mathrm{~cm}$ boca; $41,5 \mathrm{~cm}$ x $113 \mathrm{~cm}$ fundo e $15,5 \mathrm{~cm}$ altura; 8,5 litros), tendo como substrato o condicionador de floreira da Forth Jardim ${ }^{\circledR}$, composto por (casca de Pinus decomposta naturalmente e cinzas; CTC: $230 \mathrm{mmol}_{\mathrm{c}} \mathrm{kg}^{-1}$; CRA: 168\%; natureza física: farelado), no qual é recomendado pelo fabricante que seja realizada adubação (FORTHJARDIM, 2020).

Ao substrato foi adicionado adubos, formando os seguintes tratamentos T1- Substrato + NPK $\left(13-5\right.$ - 13) $\left(5 \mathrm{~g} \mathrm{~L}^{-1}\right), \mathrm{T} 2$ - Substrato + Osmocote ${ }^{\circledR} 3 \mathrm{M}$ 14-14-14 (6 g L $\left.{ }^{-1}\right)$ e T3 Substato + Osmocote ${ }^{\circledR} 3 \mathrm{M}$ 19-06-10 mini prill (sendo 1/6 menor que o tamanho padrão de Osmocote $\left.{ }^{\circledR}\right)\left(6 \mathrm{~g} \mathrm{~L}^{-1}\right)$; posteriormente a mistura foi distribuída nas jardineiras, e em seguida foram transplantadas duas mudas de I. balsamina por jardineira. A irrigação foi iniciada no mesmo dia do plantio e ocorreu de forma manual, com a adição de $800 \mathrm{~mL}$ de água por contêiner. O delineamento experimental foi inteiramente casualizado, com 3 tratamentos, 6 repetições e 2 plantas por repetição (jardineira).

\section{Avaliações}

As mudas, 42 dias após o transplante, foram avaliadas quanto a:

- Altura e diâmetro da base do caule: medida com o auxílio de uma régua graduada (da base do caule ao ápice) e paquímetro digital, respectivamente;

- Índice de clorofila foliar (ICF): analisado pelo medidor portátil eletrônico de clorofila (Clorofilômetro) ClorofiLOG CFL1030 (FALKER).

- Contagem visual do número de flores;

- Massa fresca da parte aérea (MFPA) e da raiz (MFR), pesadas em balança analítica;

- Massa seca da parte aérea (MSPA) e da raiz (MSR) das plântulas. Para as MFs, as amostras foram pesadas em balança analítica, e posteriormente colocando-as em estufa de circulação de ar forçada a $65^{\circ} \mathrm{C}$, durante 72 horas, para determinação então das MSs.

- Análise de macro (N; P; K; S; Mg; Ca) e micronutrientes $(\mathrm{Cu} ; \mathrm{Fe} ; \mathrm{Mn})$ foliares de acordo com metodologia de Malavolta, Vitti e Oliveira (1997). 
Os resultados foram submetidos à análise de variância e Teste de Tukey a 5\% de significância, utilizando o programa SISVAR para análise estatística (FERREIRA, 2008), e dos coeficientes de correlação de Pearson nas avaliações realizadas na parte aérea.

\section{RESULTADOS E DISCUSSÃO}

Pode-se observar que T2 e T3 diferem estatisticamente de T1 para os parâmetros: índice clorofila foliar e altura do caule (Tabela 1); para diâmetro de caule, com 19,95 mm sendo de maior valor e estatisticamente diferente de T1 foi para T3.

Testando Osmocote ${ }^{\circledR}$ Plus 15-4-10 nas doses 3,5; 5,3 e $7 \mathrm{~g} \mathrm{~L}^{-1}$, em New Guinea Impatiens (Impatiens hawkeri Bull.) Paradise New Red, Ostrom (2011) obteve como resultado valores entre 53,5 a 65,3 para SPAD, respectivamente, sendo esses superiores a T2 Osmocote ${ }^{\circledR} 3 \mathrm{M}$ 14-14-14 (6 g L $\left.{ }^{-1}\right)$ e T3 - Osmocote ${ }^{\circledR} 3 \mathrm{M}$ 19-06-10 mini prill $\left(6 \mathrm{~g} \mathrm{~L}^{-1}\right)$, como observado na Tabela 1. Para Pias et al., (2013) em mudas de grápia, o ICF apresentou integração com doses da adubação de Osmocote ${ }^{\circledR}$, apresentando melhores resultados com a doses do adubo de liberação lenta, no presente trabalho pode-se observar que houve semelhança nos resultados obtidos, pela diferença significativa entre os tratamentos na avaliação de ICF, visto que os melhores resultados apresentados são com adição de doses de Osmocote $^{\circledR}$, sendo T2 e T3.

Tabela 1. Índice de clorofila foliar (SPAD), altura $(\mathrm{cm})$ e diâmetro de caule de Impatiens balsamina, cultivada em 3 tratamentos, 42 dias após o transplante. Ilha Solteira / SP, 2018. Leaf chlorophyll index (SPAD), height $(\mathrm{cm})$ and stem diameter of Impatiens balsamina, grown in 3 treatments, 42 days after transplant. Ilha Solteira / SP, 2018.

\begin{tabular}{lccc}
\hline Trat. & $\begin{array}{c}\text { Índice de clorofila foliar } \\
(\text { SPAD })\end{array}$ & $\begin{array}{c}\text { Altura do caule } \\
(\mathrm{cm})\end{array}$ & $\begin{array}{c}\text { Diâmetro do caule } \\
(\mathrm{mm})\end{array}$ \\
\hline T1 & $12,93 \mathrm{~b}$ & $15,13 \mathrm{~b}$ & $4,40 \mathrm{c}$ \\
$\mathrm{T} 2$ & $51,96 \mathrm{a}$ & $52,21 \mathrm{a}$ & $16,95 \mathrm{~b}$ \\
T3 & $51,37 \mathrm{a}$ & $57,38 \mathrm{a}$ & $19,95 \mathrm{a}$ \\
\hline DMS & 4,94 & 7,49 & 2,39 \\
CV & 12,71 & 17,97 & 17,29 \\
F & $247,40^{* *}$ & $114,20^{* *}$ & $144,21^{* *}$ \\
\hline
\end{tabular}

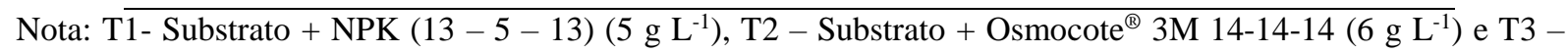
Substato + Osmocote ${ }^{\circledR} 3 \mathrm{M}$ 19-06-10 mini prill $\left(6 \mathrm{~g} \mathrm{~L}^{-1}\right)$. Médias seguidas de mesma letra na coluna não diferem entre pelo teste de Tukey a $5 \%$ de probabilidade. ns - não significativo pelo teste $\mathrm{F}$; ** - Significativo a $1 \%$ pelo teste F. Fonte: Autor, 2018. Note: T1- Substrate + NPK (13 - 5 - 13) $\left(5 \mathrm{~g} \mathrm{~L}^{-1}\right), T 2-$ Substrate + Osmocote ${ }^{\circledR} 3 M$ 14-14-14 $\left(6 \mathrm{~g} \mathrm{~L}^{-1}\right)$ and T3 - Substitute + Osmocote ${ }^{\circledR} 3 M$ 19-06-10 mini prill $\left(6 \mathrm{~g} \mathrm{~L}^{-1}\right)$. Averages followed by the same letter in the column do not differ by Tukey's test at 5\% probability. ns - non significant by the F test; ** Significant at $1 \%$ by the F test.

Fonte: Autoria própria. Own authorship.

Em trabalho com desenvolvimento de mudas de pimenta de bico (Capsicum chinense) em diferentes fertilizantes Pagliarini; Castilho e Mariano (2014) observaram que o ICF foi maior com o uso de uso de adubo de liberação lenta, em comparação ao de liberação convencional, corroborando com o presente trabalho. 
A relação altura/diâmetro do caule pode mostrar dificuldades da planta em se manter ereta e o tombamento decorrente pode resultar em morte ou deformações dos indivíduos (CUNHA et al., 2005); assim sendo, T2 e T3 tiveram que ser tutoradas. Segundo Veiling (2020) para Pote/Cuia 21 a 40, a altura de Impatiens pode variar de $16 \mathrm{~cm}$ até não ter limite, e segundo Alves (2019), variando entre 20 e $75 \mathrm{~cm}$ de altura; assim, observa-se, pela Tabela 1, o intervalo está entre 15,13 e 57,38 cm, contemplando o citado por ambos autores. Para Perry (2020), a Impatiens pode ter de 15 a $30 \mathrm{~cm}$ de altura, dependendo do espaçamento, umidade, nutrientes disponíveis e quantidade de luz solar, valores menores que os da Tabela 1, quando do uso de adubos de liberação lenta ou convencional e espaçamento de $18 \mathrm{~cm}$ entre plantas.

Com relação ao número acumulado de flores, apresentados na Figura 1, T2 teve média de 25 flores e T3 de 28; Ostrom (2011) em trabalho utilizando Osmocote Plus 15-4-10, nas doses 3,5; 5,3 e $7 \mathrm{~g} \mathrm{~L}^{-1}$, com New Guinea Impatiens (Impatiens hawkeri Bull.) Paradise New Red, obteve valores entre 23 a 28 flores na dose $7 \mathrm{~g} \mathrm{~L}^{-1}$, ou seja, no intervalo citado.

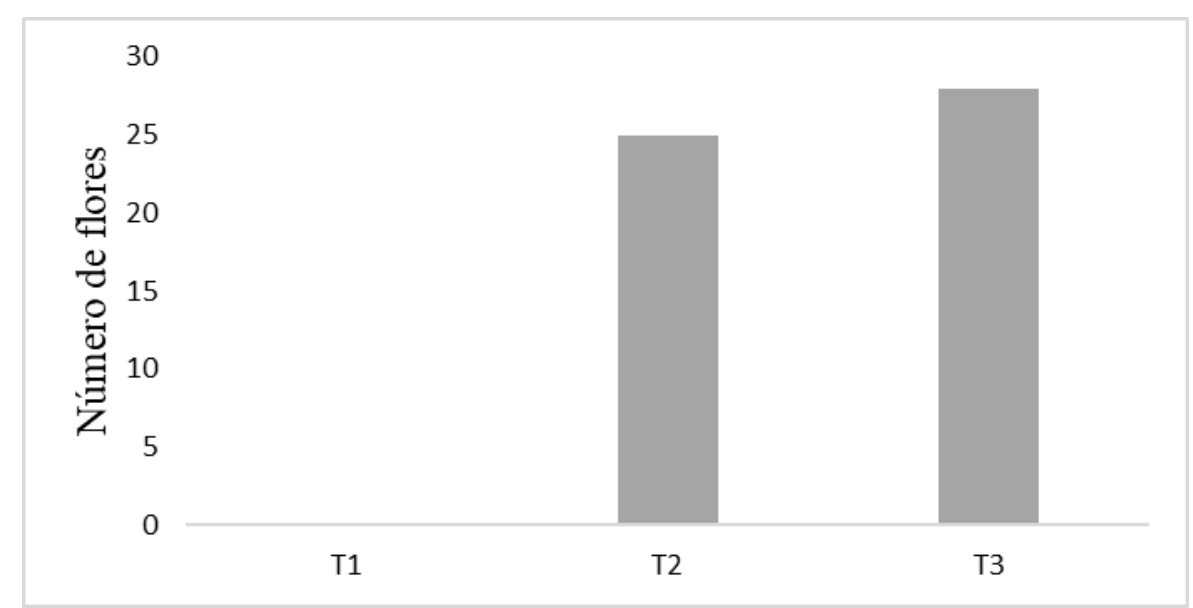

Nota: T1- Substrato + NPK (13 - 5 - 13) $\left(5 \mathrm{~g} \mathrm{~L}^{-1}\right)$, T2 - Substrato + Osmocote ${ }^{\circledR} 3 \mathrm{M} 14-14-14\left(6 \mathrm{~g} \mathrm{~L}^{-1}\right)$ e T3 Substrato + Osmocote ${ }^{\circledR} 3 \mathrm{M}$ 19-06-10 mini prill (6 g L $\left.{ }^{-1}\right)$. Fonte: Autor, 2018. Note: T1- Substrate + NPK (13 - 5 - 13) $\left(5 \mathrm{~g} \mathrm{~L}^{-1}\right), T 2$ - Substrate + Osmocote ${ }^{\circledR} 3 M$ 14-14-14 $\left(6 \mathrm{~g} \mathrm{~L}^{-1}\right)$ and T3 - Substrate + Osmocote ${ }^{\circledR}$ 3M 19-06-10 mini prill $\left(6 \mathrm{~g} \mathrm{~L}^{-1}\right)$.

Figura 1. Número de flores acumuladas por tratamentos (T1, T2, T3), de Impatiens balsamina, cultivada em 3 tratamentos, 42 dias após o transplante. Ilha Solteira/SP, 2018. Number of flowers accumulated by treatments (T1, T2, T3), of Impatiens balsamina, grown in 3 treatments, 42 days after transplantation. Ilha Solteira / SP, 2018.

Fonte: Autoria própria. Own authorship.

Na Tabela 2 observa-se que para MFR, MSPA e MSR, os tratamentos com uso de adubo de liberação lenta (T2 e T3), tiveram resultados estatisticamente diferente de T1. Para MFPA, T3 mostrou-se superior, em 25\%, a T2, possivelmente atribuído ao N (19\%), mas não refletiu nos demais resultados.

Ostrom (2011) em trabalho utilizando Osmocote Plus 15-4-10, em New Guinea Impatiens (Impatiens hawkeri Bull.) Paradise New Red, obteve 9,9 a 12,6 g MSR, superiores aos de T2 e T3 (Tabela 2). Mauta et al. (2014) utilizando fertilizante de liberação lente 15-09$12\left(3 ; 6 ; 9 ; 12 \mathrm{~g} \mathrm{~L}^{-1}\right)$, observaram que o mesmo proporcionou aumento do desenvolvimento de abacaxizeiro ornamental cultivado em vaso; Gomes et al. (2017) obtiveram efeitos positivos no incremento de altura, diâmetro de colo e massa seca de parte aérea, com o uso de adubos 
de liberação lenta em mudas de pimentão; portanto os autores mostram resultados que corroboram com o do presente trabalho.

Tabela 2. Massa fresca (MFPA) e seca (MSPA) da parte aérea, massa fresca (MFR) e seca (MSR) da raiz, em gramas (g), para Impatiens balsamina, cultivada em 3 tratamentos, 42 dias após o transplante. Ilha Solteira/SP, 2018. Fresh mass (FMAP) and dry mass (DMAP) of the aerial part, fresh mass (RFM) and dry mass (RFM) of the root, in grams ( $g$ ), for Impatiens balsamina, cultivated in 3 treatments, 42 days after transplantation. Ilha Solteira / SP, 2018.

\begin{tabular}{|c|c|c|c|c|c|c|c|c|}
\hline \multirow{2}{*}{ Tratamento } & \multicolumn{2}{|l|}{ MFPA } & \multicolumn{2}{|c|}{ MFR } & \multicolumn{2}{|c|}{ MSPA } & \multicolumn{2}{|c|}{ MSR } \\
\hline & \multicolumn{8}{|c|}{ 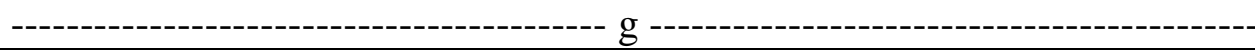 } \\
\hline 1 & 3,27 & $\mathrm{c}$ & 3,88 & $\mathrm{~b}$ & 0,20 & $\mathrm{~b}$ & 3,02 & $\mathrm{~b}$ \\
\hline 2 & 169,43 & $\mathrm{~b}$ & 46,57 & a & 12,78 & $\mathrm{a}$ & 8,15 & a \\
\hline 3 & 244,82 & $\mathrm{a}$ & 49,86 & a & 16,58 & $\mathrm{a}$ & 8,03 & $\mathrm{a}$ \\
\hline DMS & \multicolumn{2}{|l|}{56,4} & \multicolumn{2}{|c|}{13,63} & \multicolumn{2}{|c|}{3,81} & \multicolumn{2}{|c|}{0,88} \\
\hline $\mathrm{CV}$ & \multicolumn{2}{|l|}{40,44} & \multicolumn{2}{|c|}{40,68} & \multicolumn{2}{|c|}{38,61} & \multicolumn{2}{|c|}{13,8} \\
\hline $\mathrm{F}$ & \multicolumn{2}{|l|}{$57,85 * *$} & \multicolumn{2}{|c|}{$42,69 * *$} & \multicolumn{2}{|c|}{$61,01 * *$} & \multicolumn{2}{|c|}{$131,84 * *$} \\
\hline
\end{tabular}

Nota: T1- Substrato + NPK (13 - 5 - 13) $\left(5 \mathrm{~g} \mathrm{~L}^{-1}\right), \mathrm{T} 2$ - Substrato + Osmocote $^{\circledR} 3 \mathrm{M}$ 14-14-14 $\left(6 \mathrm{~g} \mathrm{~L}^{-1}\right)$ e T3 Substrato + Osmocote ${ }^{\circledR} 3 \mathrm{M}$ 19-06-10 mini prill $\left(6 \mathrm{~g} \mathrm{~L}^{-1}\right)$. Médias seguidas de mesma letra na coluna não diferem entre pelo teste de Tukey a $5 \%$ de probabilidade. ${ }^{\text {ns }}$ - não significativo pelo teste F; ** - Significativo a $1 \%$ pelo teste F. Fonte: Autor, 2018. Note: T1-Substrate + NPK $(13-5-13)\left(5 \mathrm{~g} \mathrm{~L}^{-1}\right), T 2-$ Substrate + Osmocote ${ }^{\circledR} 3 M$ 14-14-14 $\left(6 \mathrm{~g} \mathrm{~L}^{-1}\right)$ and T3 - Substrate + Osmocote ${ }^{\circledR} 3 \mathrm{M}$ 19-06-10 mini prill $\left(6 \mathrm{~g} \mathrm{~L}^{-1}\right)$. Averages followed by the same letter in the column do not differ by Tukey's test at $5 \%$ probability. ${ }^{n s}$ - non significant by the F test; ** Significant at $1 \%$ by the F test.

Fonte: Autoria própria. Own authorship.

Tabela 3. Concentrações de macro e micronutrientes da parte aérea (caule e folhas) de Impatiens balsamina, cultivada em 3 tratamentos, 42 dias após o transplante. Ilha Solteira/SP, 2018. Macro and micronutrient concentrations of the shoot (stem and leaves) of Impatiens balsamina, grown in 3 treatments, 42 days after transplantation. Ilha Solteira / SP, 2018.

\begin{tabular}{|c|c|c|c|c|c|c|}
\hline \multirow{2}{*}{ Tratamento } & $\mathrm{N}$ & $\mathrm{P}$ & $\mathrm{K}$ & $\mathrm{S}$ & $\mathrm{Mg}$ & $\mathrm{Ca}$ \\
\hline & \multicolumn{6}{|c|}{-------------------------------g kg-1 ----------------------- } \\
\hline 1 & $13,6 b$ & $4,2 \mathrm{c}$ & $19,47 b$ & $18,6 \mathrm{a}$ & $6,60 \mathrm{a}$ & $23,19 \mathrm{a}$ \\
\hline 2 & $33,0 \mathrm{a}$ & $10,9 \mathrm{a}$ & $33,64 \mathrm{a}$ & $8,4 \mathrm{~b}$ & $4,93 \mathrm{a}$ & $23,23 \mathrm{a}$ \\
\hline 3 & $35,9 \mathrm{a}$ & $7,1 b$ & $35,26 \mathrm{a}$ & $6,9 \mathrm{~b}$ & $6,01 \mathrm{a}$ & $29,24 \mathrm{a}$ \\
\hline DMS & 7,4 & 2,8 & 3,87 & 1,6 & 2,82 & 14,31 \\
\hline $\mathrm{CV}$ & 10,8 & 15,03 & 43,16 & 5,82 & 19,26 & 22,64 \\
\hline $\mathrm{F}$ & $50,25 * *$ & $27,38 * *$ & $1,4^{\mathrm{ns}}$ & $281,36 * *$ & $1,7^{\mathrm{ns}}$ & $1,11^{\mathrm{ns}}$ \\
\hline \multirow{2}{*}{ Tratamento } & \multicolumn{2}{|c|}{$\mathrm{Cu}$} & \multicolumn{2}{|c|}{$\mathrm{Fe}$} & \multicolumn{2}{|c|}{$\mathrm{Mn}$} \\
\hline & \multicolumn{6}{|c|}{---------------- $\mathrm{mg} \mathrm{kg}^{-1}$---------------- } \\
\hline 1 & \multicolumn{2}{|c|}{$10.67 \mathrm{a}$} & \multicolumn{2}{|c|}{$372,00 \mathrm{a}$} & \multicolumn{2}{|c|}{$160,00 \mathrm{a}$} \\
\hline 2 & \multicolumn{2}{|c|}{$17,33 \mathrm{a}$} & \multicolumn{2}{|c|}{$202,67 b$} & \multicolumn{2}{|c|}{$102,00 \mathrm{~b}$} \\
\hline 3 & \multicolumn{2}{|c|}{$18,00 \mathrm{a}$} & \multicolumn{2}{|c|}{$143,67 b$} & \multicolumn{2}{|c|}{$104,33 b$} \\
\hline DMS & \multicolumn{2}{|c|}{13,70} & \multicolumn{2}{|c|}{112,18} & \multicolumn{2}{|c|}{51,68} \\
\hline $\mathrm{CV}$ & \multicolumn{2}{|c|}{35,65} & \multicolumn{2}{|c|}{18,69} & \multicolumn{2}{|c|}{16,88} \\
\hline $\mathrm{F}$ & \multicolumn{2}{|c|}{$1,65^{\mathrm{ns}}$} & \multicolumn{2}{|c|}{$21,04 * *$} & \multicolumn{2}{|c|}{$7,61 *$} \\
\hline
\end{tabular}

Nota: T1- Forth Plantio $13-5$ - $13\left(5 \mathrm{~g} \mathrm{~L}^{-1}\right), \mathrm{T} 2$ - Osmocote ${ }^{\circledR} 3 \mathrm{M}$ 14-14-14 $\left(6 \mathrm{~g} \mathrm{~L}^{-1}\right)$ e T3 - Osmocote ${ }^{\circledR} 3 \mathrm{M} 19$ 06-10 mini prill $\left(6 \mathrm{~g} \mathrm{~L}^{-1}\right)$. Médias seguidas de mesma letra na coluna não diferem entre pelo teste de Tukey a $5 \%$ de probabilidade. ${ }^{\text {ns }}$ - não significativo pelo teste $\mathrm{F} ;{ }^{* *}$ - Significativo a $1 \%$ pelo teste F. Note: T1- Forth 
Planting 13 - 5 - 13 (5 $\left.\mathrm{g} \mathrm{L} \mathrm{L}^{-1}\right)$, T2 - Osmocote ${ }^{\circledR}$ 3M 14-14-14 (6 $\left.\mathrm{g} \mathrm{L}^{-1}\right)$ and T3 - Osmocote ${ }^{\circledR}$ 3M 19-06-10 mini prill $\left(6 \mathrm{~g} \mathrm{~L}^{-1}\right)$. Averages followed by the same letter in the column do not differ by Tukey's test at $5 \%$ probability. ns - non significant by the F test; ** - Significant at $1 \%$ by the F test.

Fonte: Autoria própria. Own authorship.

Em trabalho com New Guinea Impatiens, Judd e Cox (1995) observaram que quanto maior a dose de $\mathrm{N}$ no adubo, menor foi MS da parte aérea da planta, não ocorrendo o mesmo neste trabalho, visto que o de adubo liberação lenta em $\mathrm{T} 3 \mathrm{~N}=19 \%$ e o de liberal convencional $\mathrm{N}=13 \%$.

Os resultados da análise foliar da parte aérea (caule e folhas), observa-se o efeito do adubo de liberação lenta e convencional em N, P e K em comparação com a testemunha; os resultados corroboram também com os vistos nas Tabelas 2 e 3 .

Para Altland (2019), os teores de nutrientes encontrados em diversas espécies de plantas saudáveis devem ser, em $\mathrm{g} \mathrm{kg}_{-}{ }^{1}: \mathrm{N}=40, \mathrm{P}=5, \mathrm{~K}=15, \mathrm{Ca}=15, \mathrm{Mg}=5, \mathrm{~S}=2,5$, valores esses discrepantes no encontrado no presente trabalho, exceto para $\mathrm{Mg}$. Apesar $\mathrm{T} 3$ ter maior concentração de $\mathrm{N}$ no adubo utilizado (19\%) do que T2 (14\%), isso não refletiu estatisticamente na análise apresentada na Tabela 4.

Tabela 4. Coeficientes de correlação de Pearson das avaliações realizadas na parte aérea de Impatiens balsamina, cultivada em 3 tratamentos, 42 dias após o transplante. Ilha Solteira/SP, 2018. Pearson's correlation coefficients of the evaluations performed in the Impatiens balsamina shoot, cultivated in 3 treatments, 42 days after transplantation. Ilha Solteira/SP, 2018.

\begin{tabular}{l|ccccc}
\hline \multicolumn{1}{c|}{ Variáveis } & N foliar & Clorofila & Altura & Diâmetro & $\begin{array}{c}\text { Massa fresca } \\
\text { Parte aérea }\end{array}$ \\
\hline Clorofila & $0,9668^{* *}$ & & & & \\
Altura & $0,9316^{* *}$ & $0,9553^{* *}$ & & & \\
Diâmetro & $0,9370^{* *}$ & $0,9479 * *$ & $0,9919 * *$ & & \\
Massa fresca parte aérea & $0,9489 * *$ & $0,9822^{* *}$ & $0,9907 * *$ & $0,9865 * *$ & \\
Massa seca parte aérea & $0,9675^{* * *}$ & $0,9642 * *$ & $0,9749 * *$ & $0,9821 * *$ & $0,9820 * *$ \\
\hline
\end{tabular}

Nota: ** - significativo a 1\%; Note: ** - significant at $1 \%$.

Fonte: Autoria própria. Own authorship.

Em trabalho utilizando Osmocote Plus 15-4-10 / $7 \mathrm{~g} \mathrm{~L}^{-1}$ com New Guinea Impatiens (Impatiens hawkeri Bull.) Paradise New Red, Ostrom (2011) verificou-se concentrações, em g $\mathrm{kg}^{-1}$ de $\mathrm{N}=32,9, \mathrm{P}=3,8, \mathrm{~K}=16,0, \mathrm{Ca}=10,6, \mathrm{Mg}=10,2$ e $\mathrm{s}=7,5$, sendo portanto semelhante somente para $\mathrm{N}$ (Tabela 3); e em mg kg-1, de $\mathrm{Cu}=6,42, \mathrm{Fe}=71,73, \mathrm{Mn}=67,9$ e $\mathrm{Zn}=53,17$, onde todos foram inferiores aos encontrados na Tabela 3; deve-se levar em consideração que o substrato utilizado pelo referido autor foi turfa e perlite (7:3).

Infere-se que os maiores valores absolutos observados nas Tabelas 1, 2 e 3 para T3 (Condicionador + Osmocote ${ }^{\circledR} 3 \mathrm{M}$ 19-06-10 mini prill $/ 6 \mathrm{~g} \mathrm{~L}^{-1}$ ) podem ser devidos a quantidade ao N do adubo, visto que Hammer (1992) e de acordo com Romero, Taber e Gladon (2006), a produção de plantas de Impatiens walleriana requer otimização do regime nutricional durante o crescimento, especialmente com relação ao $\mathrm{N}$. 
A correlação de Pearson das avaliações realizadas na parte aérea de Impatiens balsamina consta na Tabela 4. Segundo Dancey e Reidy (2005) existe uma relação classificada de forte quando $r=0,70$ até 1, e, segundo Figueiredo Filho e Silva Júnior (2009) quanto mais perto de 1 maior é o grau de dependência estatística linear entre as variáveis. Assim, as variáveis analisadas, sendo $\mathrm{N}$ foliar, clorofila, altura, diâmetro e MFPA, estão fortemente correlacionadas entre si, sendo esta correlação linear.

\section{CONCLUSÃO}

Conclui-se que melhores resultados encontrados nas avaliações fisiológicas e morfológicas de Impatiens balsamina, foram obtidos com uso de adubo de liberação lenta, com ênfase no tratamento T3 - Substato + Osmocote ${ }^{\circledR} 3 \mathrm{M} 19-06-10$ mini prill $\left(6 \mathrm{~g} \mathrm{~L}^{-1}\right)$.

\section{AGRADECIMENTOS}

As empresas Topseed ${ }^{\circledR}$ e Forthjardim ${ }^{\circledR}$, e a Camila Morais Ramos da Silva, por ceder as mudas utilizadas na condução deste do trabalho.

\section{REFERÊNCIAS BIBLIOGRÁFICAS}

AGRISTAR. Blue line flores: Balsamina Camélia Sortida. Campinas: AGRISTAR, 2020. Disponível em: https://agristar.com.br/topseed-garden/blue-line-flores/balsamina-cameliasortida/2452030. Acesso em: 20 mar. 2020.

ALVES, M. Beijo-de-frade é uma flor vistosa e de fácil propagação. [S. l.]: Agro 2.0, 2019. Disponível em: https://agro20.com.br/beijo-de-frade/. Acesso em: 20 mar. 2020.

ALTLAND, J. Plant Nutrition Diagnosis. $\left[\begin{array}{llll}S . & l .: & \mathrm{s} . & \mathrm{n} .\end{array}\right]$, 2019. Disponível em: https://pnwhandbooks.org/plantdisease/pathogen-articles/nonpathogenic-phenomena/plantnutrition-diagnosis. Acesso em: 20 set. 2019.

BACKES, C.; FERNANDES, F. M.; KROHN, N. G.; LIMA, C. P.; KIIHL, T. A. M. Produção de pimenta ornamental em função de substratos e doses de adubação com fertilizantes de liberação lenta e tradicional. Scientia Agraria Paranaensis, Acrelândia, v. 6, n. 1-2, p.67-76, 2007. Disponível em: http://erevista.unioeste.br/index.php/scientiaagraria/article/viewFile/2042/1613. Acesso em: 20 mar. 2020.

BRAINER, M. S. C. Quando nem tudo são flores, a floricultura pode ser uma alternativa. Caderno Setorial ETENE, ano 3, n. 42, p.1-17, 2018. Disponível em: www.bnb.gov.br/documents/80223/4049480/42_Flores_2018.pdf/022d87e8-c8db-1a98-b760419661cf4e25. Acesso em: 20 set. 2019. 
BRASIL. Câmara dos Deputados. Projeto de Lei $n^{\circ} 4485$, de 2019. Institui a Política Nacional de Incentivo à Cultura de Flores e de Plantas Ornamentais de Qualidade. Brasília, DF, 2019. Disponível em: https://www25.senado.leg.br/web/atividade/materias//materia/138117. Acesso em: 14 abr. 2020.

CUNHA, A. O.; ANDRADE, L. A.; BRUNO, R. L. A.; SILVA, J. A. L.; SOUZA, V. C. Efeitos de substratos e das dimensões dos recipientes na qualidade das mudas de Tabebuia impetiginosa (Mart. Ex D.C.) Standl. Revista Árvore, Viçosa, v. 29, n. 4, p.507-516, 2005.

DANCEY, C.; REIDY, J. Estatística sem matemática para psicologia: usando SPSS para Windows. Porto Alegre: Artmed, 2006. 177 p.

FERREIRA, D. F. SISVAR: Um programa para análises e ensino de estatística. Revista Symposium, Lavras, v. 6, n. 2, p.36-41, 2008.

FIGUEIREDO FILHO, D. B.; SILVA JÚNIOR, J. A. Desvendando os mistérios do coeficiente de correlação de Pearson (r). Revista Política Hoje, Recife, v. 18, n. 1, p.115-146, 2009.

FORTH JARDIM. Forth condicionador floreira. [S. l.: s. $n$.$] , 2020. Disponível em:$ https://forthjardim.com.br/produto/forth-condicionador-floreiras. Acesso em: 20 mar. 2020.

FURTINI NETO, A. E.; BOLDRIN, K. V. F.; MATTSON, N. S. Nutrition and quality in ornamental plants. Ornamental Horticulture, Campinas, v. 21, n. 2, p.139-150, 2015.

GOMES, E. N. GEMIN, L. G., MUZEKA, G., ROSSA, Ü. B., \& WESTPHALEN, D. J. Fertilizante de liberação lenta no desenvolvimento inicial de mudas de pimentão e berinjela. Cultivando o Saber, Cascavel, v. 10, n. 2, p.166-178, 2017. Disponível em: https://www.fag.edu.br/upload/revista/cultivando_o_saber/59a5b4a59c45f.pdf. Acesso em: 20 mar. 2020.

HAMMER, P. A. Other Flowering Pot Plants. In: _ _. Introduction to Floriculture. 2. ed. [s.1.]: Academic Press, 1992. cap. 19, p. 489. Disponível em: http://dx.doi.org/10.1016/B9780-12-437651-9.50024-5. Acesso em:12 jul. 2020.

JUDD, L.; COX, D. New guinea impatiens: watch out for soluble salts. Disponível em: https://ag.umass.edu/greenhouse-floriculture/fact-sheets/new-guinea-impatiens-watch-out-forsoluble-salts. Acesso em: 20 mar. 2020.

LATIMER, J. G. Feature, mechanical conditioning for control of growth and quality of vegetable transplants. HortScience, Alexandria, v. 26, n. 12, p.1456-1461, 1991.

MALAVOlTA, E.; VITTI, G. C.; OLIVEIRA, S. A. Avaliação do estado nutricional das plantas: princípios e aplicações. 2. ed. Piracicaba: Potafos, 1997. 319 p.

MAUTA, D. S.; HAERROTH, F. J.; SERRANO, L. A. L.; FEITOSA, M. M.; OLIVEIRA, M. M. T. Adubo de Liberação Controlada (NPK 5-09-12) na Produção de Abacaxizeiro Ornamental em Vaso. 2014. Disponível em: https://www.alice.cnptia.embrapa.br/alice/bitstream/doc/1006961/1/RES14022.pdf. Acesso em 20 set. 2019. 
MOREIRA, M. L.; BENTO, C. S. Levantamento da produção de flores e plantas ornamentais no caparaó capixaba. In: SEMANA ACADÊMICA DO CURSO DE AGRONOMIA DO CCAE/UFES, 30, 2018, Vitória. Anais [...] Vitória: UFES, 2018. Disponível em: http://periodicos.ufes.br/SEAGRO/article/view/20475/14335. Acesso em: Acesso em 01mar. 2019.

OSTROM, A. K. Comparing the effect of controlled-release, slow-release, and watersoluble fertilizers on plant growth and nutrient leaching. 2011. $131 \mathrm{f}$. Thesis (Master of Science in the Graduate School) - The Ohio State University, Ohio, 2011. Disponível em: https://etd.ohiolink.edu/!etd.send_file?accession=osu1299641954\&disposition=inline. Acesso em: 20 mar. 2020.

PAGLIARINI, M. K.; CASTILHO, R. M. M.; MARIANO, F. A. C. Desenvolvimento de mudas de pimenta de bico em diferentes fertilizantes. Revista Brasileira de Horticultura Ornamental, Campinas, v. 20, n. 1, p.35-42, 2014.

PERRY, L. Impatiens. Burlington: University of Vermont Extension Department of Plant and Soil Science, 2020. Disponível em: https://pss.uvm.edu/ppp/articles/impatien.html. Acesso em: 20 mar. 2020.

PIAS, O. D. C.; CANTARELL, E. B.; BERGHETTI, J.; LESCHEWITZ, R.; KLUGE, E. R.; SOMAVILLA, L. Doses de fertilizante de liberação controlada no índice de clorofila e na produção de mudas de grápia. Pesquisa Florestal Brasileira, Colombo, v. 33, n. 73, p.19-26, 2013.

RIVAS, L. Setor de floricultura crescerá cinco vezes mais do que o PIB brasileiro em 2018. Guaíba: Rádio Guaíba, 2018. Disponível em: https://guaiba.com.br/2018/09/10/setorde-floricultura-crescera-cinco-vezes-mais-do-que-o-pib-brasileiro-em-2018/. Acesso em: 20 set. 2019.

ROMERO, F. R.; TABER, H. G.; GLADON, R. J. Nitrogen Source and Concentration Affect Growth and Performance of Bedding-Plant Impatiens. Journal of Plant Nutrition, London, v. 29, n. $7, \quad$ p.1315-1326, 2006. Disponível em: https://www.tandfonline.com/doi/full/10.1080/01904160600767658?scroll=top\&needAccess $=$ true. Acesso em 14 abril 2020.

SHARMA, G. C. Controlled-release fertilizers and horticultural applications. Scientia Horticulturae, Amsterdam, v.11, n. 2, p.107-129, 1979.

TANAKA, M. K.; CHAVES, D. V. Noções básicas para a produção de flores. Belo Horizonte: [s. n.], 2019. Disponível em: https://www.bioneem.com.br/nocoes-basicas-paraproducao-de-flores-POR-DAZIO-VILELA.pdf. Acesso em: 14 abr. 2020.

VEILING. Impatiens e sunpatiens de vaso. Holambra: Veiling, 2020. Disponível em: http://veiling.com.br/uploads/padrao/impatiens-fv.pdf. Acesso em: 14 abr. 2020. 